\title{
SUPER-EDDINGTON ACCRETION IN BLACK HOLE X-RAY BINARIES
}

\author{
M. R. Truss ${ }^{1}$ \\ RESUMEN
}

Presento simulaciones hidrodinámicas de rnuy alta resolución de discos de acreción en binarias de rayos $\mathrm{X}$ de agujero negro cuya acreción se encuentra cerca del límite de Eddington, incluyendo los efectos de irradiación y pérdida de masa. Muestro que la variabilidad extrema de fuentes tales como GRS $1915+105$ se puede explicar dentro del marco de transferencia de masa en un disco turbulento, parcialmente irradiado.

\section{ABSTRACT}

I present very high resolution hydrodynamical simulations of accretion discs in black hole X-ray binaries accreting near the Eddington limit, including the effects of disc irradiation and mass loss. I show that the extreme variability displayed by sources such as GRS $1915+105$ can be explained within the framework of an outburst in a partially irradiated, turbulent disc.

Key Words: BINARIES: CLOSE - BLACK HOLE PHYSICS - INSTABILITIES - X-RAYS: STARS

\section{INTRODUCTION}

The soft X-ray transients (SXTs) are low-mass Xray binaries in which gas is accreted via a disc onto a black hole or a neutron star. The accretion process is treme'ndously efficient, and is the driving force behind the outbursts that are the signature of SXTs. There has been renewed interest in these objects following reccut observations of ultra-luminous X-ray sources (ULXs) in nearby galaxies. These sources have luminosities in excess of the Eddington linit for a stellar mass accretor. One possibility is that ULXs contain much more massive accretors, intermediatemass black holes with $M_{1} \sim 10^{2}-10^{4} \mathrm{M}$. (Colbert \& Mushotzky 1999; Ebisuzaki et al. 2001). An alternative possibility is that the majority of ULXs are associated with SXTs. King et al. (2001) have pointed out that the observations can be explained if the X-rays from an object accreting near, but not beyond the Eddington limit are emitted anisotropically. Begelman (2002) has also suggested a mechanism in which super-Eddington accretion is allowed in a magnetised accretion disc. In this proceeding I present numerical calculations of accretion discs in long-period $\left(P_{\text {orb }} \sim\right.$ days $)$ SXTs, accreting near the Eddington limit .

An outstanding candidate for super-Eddington accretion is the galactic microquasar GRS 1915+105, a transient that has been in a continuous state of outburst for the past ten years with no sign of a decline (Figure 1). The mass of the accretor has been found to be $14 \pm 4 \mathrm{M}_{\odot}$ (Greiner et al. 2001) and given the extremely high $\mathrm{X}$-ray luminosity $\left(L_{\mathrm{X}} \sim\right.$

\footnotetext{
${ }^{1}$ University of St Andrews, UK.
}

$10^{40} \mathrm{erg} \mathrm{s}^{-1}$ ), it is likely that the black hole is accreting near the Eddington limit. There are some tantalising clues to the factors that influence the accretion disc. The outburst is lasting much longer than those of other SXTs, which decline after a year or so. The orbital period is the longest of any known SXT: $33.5 \mathrm{~d}$, so the accretion disc must be extremely large. For an inferred secondary mass of $1.2 \mathrm{M}_{\odot}$, the mass ratio of donor to accretor must be small $(q \sim 0.08)$, hence the disc can become tidally unstable in the same way as in the SU UMa class of cataclysmic variables. It has already been shown in hydrodynamic simulations of SU UMas that the tidal instability prolongs the outburst; there is nothing to stop this process operating in SXTs (Truss et al. 2001; 2002).

\section{SIMULATING OUTBURSTS IN IRRADIATED DISCS}

I use a two-dimensional Smoothed Particle Hydrodynamics (SPH) code to simulate the gas flow in an accretion disc in the Roche potential of a close binary star. SPH is a Lagrangian method in which a fluid is described by a set of particles moving with the local fluid velocity. I include a treatment of the hydrogen ionization instability and a simple model for the effects of irradiation of the disc by the X-rays emitted from the accretor. I assume that the X-ray irradiation is sufficient to keep the disc in an ionised, high viscosity state out to a certain radius. The radius of the irradiated region of the disc is determined by the magnitude of the central accretion rate (King 


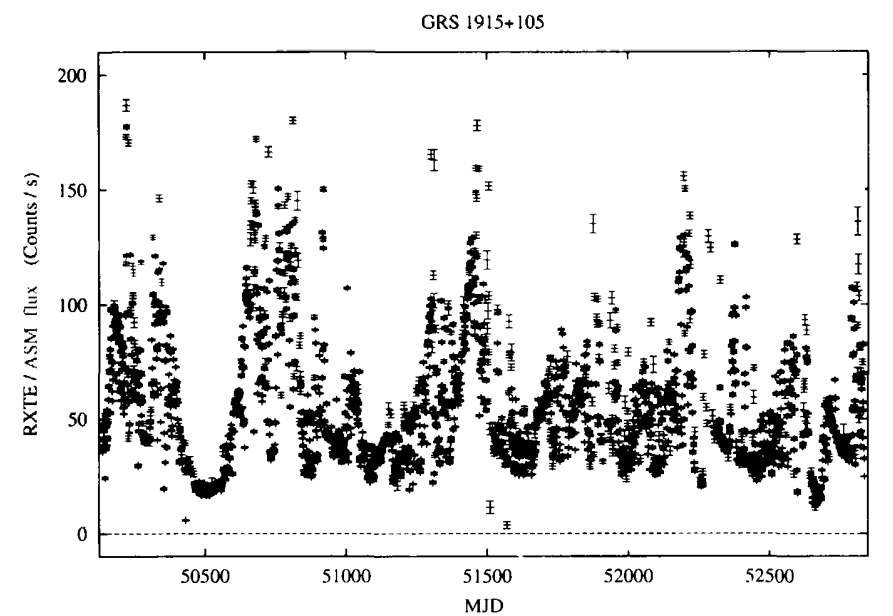

Fig. 1. RXTE ASM X-ray one-day average light curve of GRS $1915+105$.

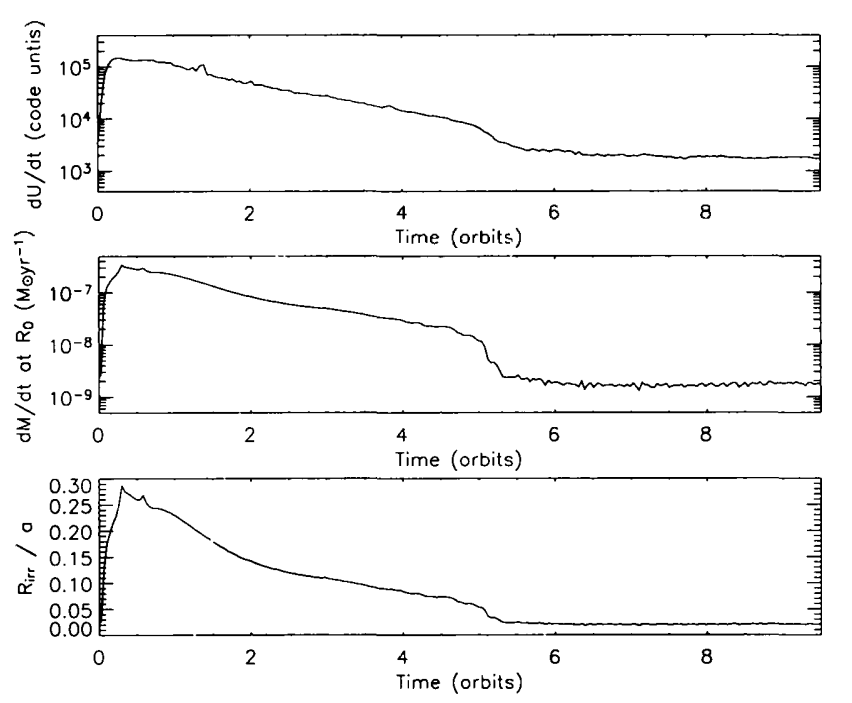

Fig. 2. Time-dependence of total energy dissipation rate (top), central accretion rate (centre) and $R_{\mathrm{X}}$ as a fraction of binary separation, a (bottom) for simulation 1 , in which no limit was placed on the accretion rate or the radius of the irradiated region.

\& Ritter 1998):

$$
R_{\mathrm{X}} \propto\left(\eta \frac{d M_{1}}{d t}\right)^{\frac{1}{2}}
$$

where $\eta$ is the accretion efficiency. Therefore, there is a maximum radius $R_{\mathrm{X}}$ can take which corresponds to accretion at (and beyond) the Eddington rate. The local accretion rate can be calculated from the local radial velocity, and since the local luminosity $L(r) \propto r^{-1} d M(r) / d t$, it is possible to reject particles from the gas flow wherever $L(r)>L_{\text {Eddington. }}$.

\section{RESULTS}

Three simulations were performed of an accretion disc in a binary system with the parameters of GRS $1915+105\left(q=0.08 ; P_{\text {orb }}=33.5 \mathrm{~d} ; M_{1}=14 M_{(\odot)}\right)$.

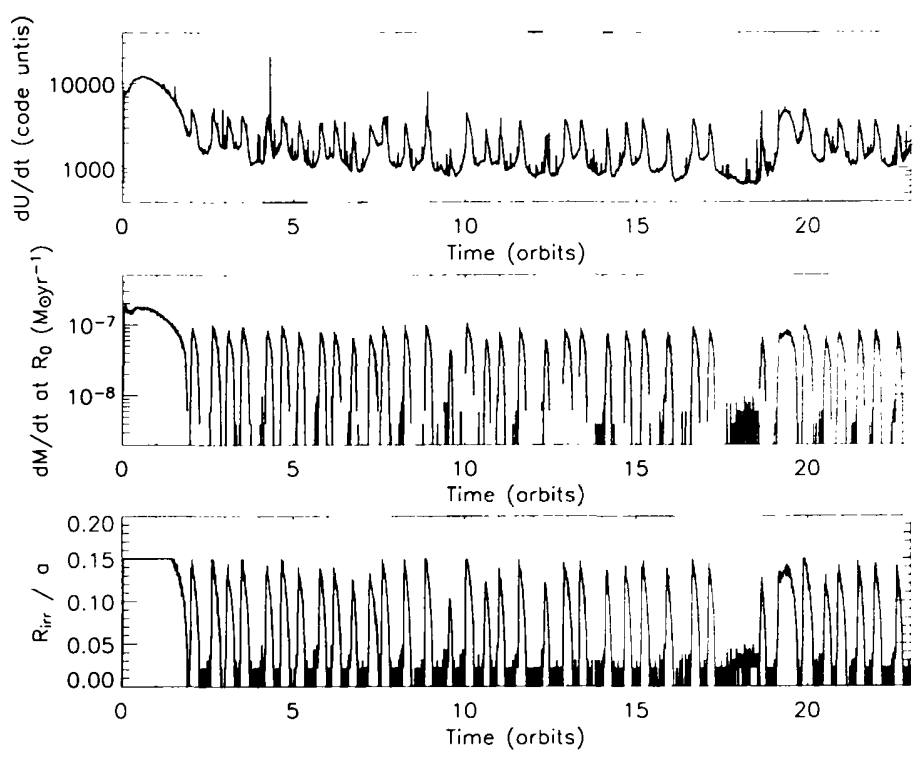

Fig. 3. As Figure 2, but for simulation 2, in which $R_{\mathrm{X}}$ was limited to a maximum value whenever $L\left(R_{\mathrm{in}}\right) \geq$ $L_{\text {Eddington }}$.
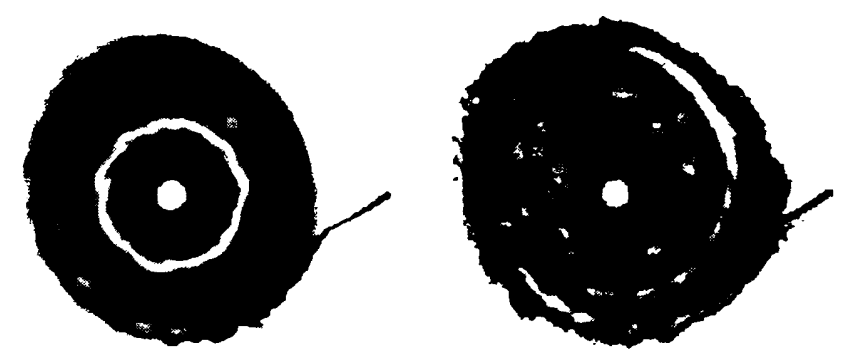

Fig. 4. Accretion disc surface density (pale colours high) for simulation 2 at $\mathrm{t}=0.3$ (left) and $\mathrm{t}=5$ (right).

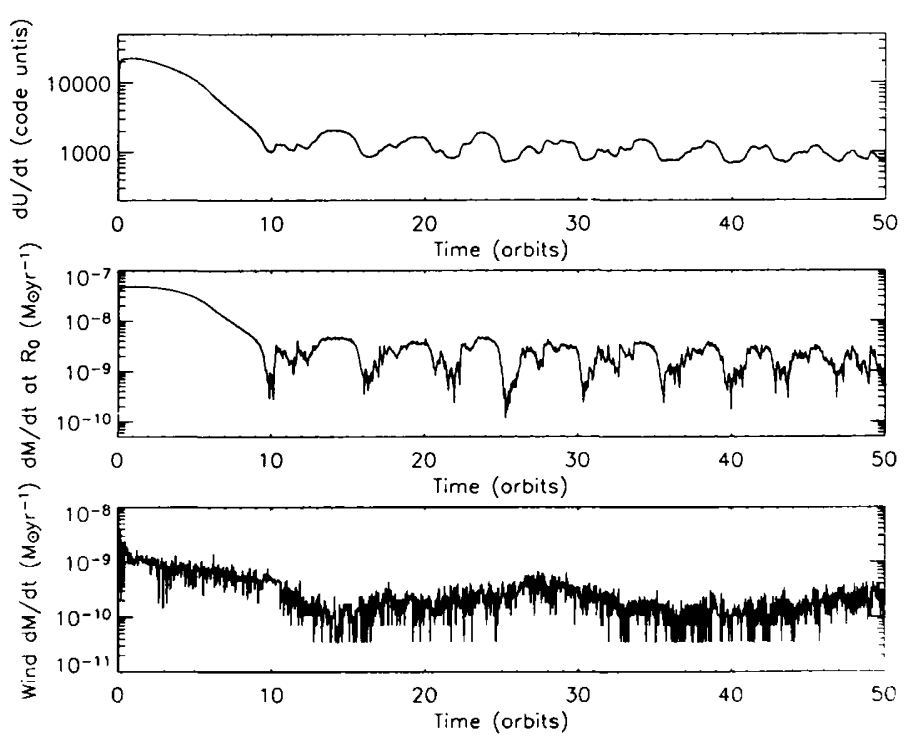

Fig. 5. Time-dependence of total ('ne'rgy dissipation rate (top), central accretion rate (centre) and local mass his rate (bottom) for simulation3, where mass was removed locally from the flow whenever $L(r)>L_{\text {Eddington }}$. 
The disc was built up from seratch by injecting particles from the inner Lagraugian point at a rate $-\dot{M}_{2}=10^{-8} \mathrm{MI}, \mathrm{yr}^{-1}$. This rate is comsistent with theories of mass-transing from an evolved serendary in a binary with $P_{\text {orb }}=33.5 \mathrm{~d}$. When the surface density muwhere in the disc crosses the critical threshold for the disc instability. the ShakuraSunyarev viscesily paraneter. $\alpha$, and the local sound speed are incrased and an outburst is triggered. The initial conditions for all three simulations : are identical. with a number of SPH particles $N_{0}=$ 139,603. The details of ark simulation ane as follows: Simulation 1: No limit was placed on the inner accrotion rate or $R_{x}$; Simulation $2: R_{X}$ was limiterl to a maximum value whenever $L\left(R_{\mathrm{in}}\right)>L_{\text {Edddington }}$; Simulation 3: mass was removerd locally from the flow whenever $L(r)>L_{\text {fistdington }}$.

In case 1 , the cut ire dise rapidly becomes irradiated and a large fraction of the disc mass is deposited onto the black hole. The top panel of Figure 2 shows the variation in contral arceretion rate with time during the simulation. The onthurst is extrencly simple: an exponential dicrline (the classic 'flat-top' to the outburst) followed by a more rapid cut-off as the irradiated portion recedes back toward the hole.

Case 2 shows much more complex variability. Here, $R_{\mathrm{X}}$ is limited to a maximum value well inside the outer edge of the disc. The flow through the hot/cold boundary is not steady, leading to a very complex density structure in the hot region (Figure 4) and a highly variable accretion rate onto the hole. This is reflected in the light curve (Figure 3). Meanwhile, the surface density of the gas in the outer. unirradiated region of the dise crosses the disc instability threshold and these parts are also heited. As gas is accreted the dise odge expannels and is pushed right ont, through the $3: 1$ tidal resonance radius to the maximmun tidal rartius. The orbits near the edge are highly non-circular: this adds another layer of complexity to the flow (Figure 4), and the resultant variability can be sustained for a very long period of time.

During the initial phase of the ontburst when gas piles up at the irradiated boundary, high surface densities a: $: d$ super-Eddington local aceretion rates may be achiered ( $\sim 3.5 \times 10^{-6} \mathrm{M} \ldots \mathrm{r}^{\cdots 1}$ at the boundary). In case 3, a significant fraction of mass is lost in this way (i.c. in a wind from the disc). and although the variat)ility in the light curve (middle panel of Figure j) is simpler than in case 2 (because much of the mass does not end up at the black hole) a degree of variability can still be sustained in the central accretion rate.

\section{CONCLUSIONS}

For accretion onto a black hole at the Eddington rate, the flow of gas in the disc through the irradiated/non-irradiated boundary is highly variable. The variability is consistent with that observed in the X-ray light curve of GRS1915+105 (varying on time-scales of weeks to months). Despite these fluctuations. in case 2 above, the disc quickly maintains a quasi-steady state with $\left\langle\dot{M}_{1}>\sim-\dot{M}_{2}\right.$. It is the high mass transfer rates from the secondary star found in long period SXTs that allow accretion near the Eddington limit to be sustained in this way.

There exists a localised spike in surface density and accretion rate at the boundary for a short time after the onset of an outburst. This can drive mass loss from the surface of the disc if the shock is close enough to the black hole (i.e. when $L\left(R_{\mathrm{X}}\right)>$ $\left.L_{\text {Eddington }}\right)$, and produce further variability in the central accretion rate. The time-scale of this variability will scale with the distance of the shock from the black hole.

I am grateful to the UK Particle Physics and Astronomy Research Council for a Postdoctoral Fellowship, and to Graham Wynn and Andrew King for helpful discussions. The simulations were performed on the UK Astrophysical Fluids Facility (UKAFF). The RXTE light curve was provided by the RXTE/ASM team at MIT and RXTE SOF and GOF at NASA Goddard S.F.C.

\section{REFERENCES}

Colbert E.J.M., Mushotzky R.F. 1999, ApJ, 519, 89

Ebisuzaki T. et al. 2001, ApJ, 562, L19

Greiner J., Cuby J.G., McCaughrean M.J. 2001, Nature, 414,422

King A.R., Davies M.B., Ward M.J., Fabbiano G., Elvis ‥ 2001. ApJ, 552, L109

King A.R., Ritter H. 1998, MNRAS, 293, L42

Makishima et al. 2000, ApJ, 535, 632

Truss M.R., Murray J.R., Wymn G.A. 2001, MNRAS, 324. L1

Truss M.R., Wymn G.A., Murray J.R., King A.R. 2002, MNRAS, 337. 1329

School of Physics if Astronomy: Inirensity of St Andrews. North Haugh, St Andrews, Fife, KY16 9SS, UK

(nint2ast-androws.ac.uk). 